\title{
Pim1 kinase positively regulates myoblast behaviors and skeletal muscle regeneration
}

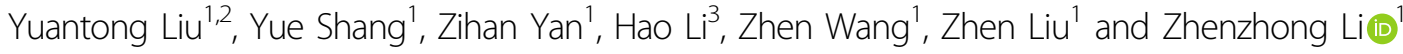

\begin{abstract}
Adult skeletal muscle regeneration after injury depends on normal myoblast function. However, the intrinsic mechanisms for the control of myoblast behaviors are not well defined. Herein, we identified Pim1 kinase as a novel positive regulator of myoblast behaviors in vitro and muscle regeneration in vivo. Specifically, knockdown of Pim1 significantly restrains the proliferation and accelerates the apoptosis of myoblasts in vitro, indicating that Pim 1 is critical for myoblast survival and amplification. Meanwhile, we found that Pim1 kinase is increased and translocated from cytoplasm into nucleus during myogenic differentiation. By using Pim1 kinase inhibitor, we proved that inhibition of Pim1 activity prevents myoblast differentiation and fusion, suggesting the necessity of Pim1 kinase activity for proper myogenesis. Mechanistic studies demonstrated that Pim1 kinase interacts with myogenic regulator MyoD and controls its transcriptional activity, inducing the expression of muscle-specific genes, which consequently promotes myogenic differentiation. Additionally, in skeletal muscle injury mouse model, deletion of Pim1 hinders the regeneration of muscle fibers and the recovery of muscle strength. Taken together, our study provides a potential target for the manipulation of myoblast behaviors in vitro and the myoblast-based therapeutics of skeletal muscle injury.
\end{abstract}

\section{Introduction}

Skeletal muscle has an ability of regeneration after injury due to its intrinsic stem cell reserve ${ }^{1}$. Skeletal muscle stem cells, also known as muscle satellite cells (SCs), reside between the plasma membrane and basal lamina in a quiescent state $^{2}$. Once the skeletal muscle is damaged, the quiescent SCs are immediately activated to generate transient amplifying precursors called myoblasts $^{3}$, which further differentiate and fuse either to form new multinucleated muscle fibers or to repair damaged parts of existing muscle fibers ${ }^{1}$. The regulation of these steps is closely associated with the sequential activation of a series of myogenic regulatory factors in SCs. Quiescent SCs highly express the transcriptional factor Pax7, while

\footnotetext{
Correspondence: Zhenzhong Li (zli@sdu.edu.cn)

'Department of Anatomy, Shandong University School of Basic Medical

Sciences, Jinan 250012, China

2Department of Spine Surgery, Shenzhen People's Hospital, Second Clinical

Medical College of Jinan University, Shenzhen 518020, China

Full list of author information is available at the end of the article.

Edited by D. Aberdam
}

the activated SCs follow the specification to the myogenic lineage and begin to express Myf5, MyoD, myogenin, and MRF4, determining the entry of myoblast into the myogenic differentiation program, which eventually fuse with injured myofibers to accomplish regeneration ${ }^{4}$. Meanwhile, a part of proliferating myoblasts downregulates MyoD and maintains Pax7, returning the quiescent state and replenishing the stem cell pool ${ }^{5}$.

However, the intrinsic mechanisms that regulate these morphological and molecular events of muscle SCs remain poorly understood. So that in some special cases, such as extensive muscle trauma and severe myopathies, skeletal muscle will have difficulty initiating the regeneration program due to the exhaustion or functional defect of muscle SCs. Although some experimental studies and clinical trials based upon intramuscular myoblasts transplantation have shown encouraging results ${ }^{6-9}$, these outcomes are still of limited benefit ascribing to the poor ability of transplanted myoblasts to survival, expansion, and migrate ${ }^{2,10-12}$. Therefore, exploring the

\section{(c) The Author(s) 2019}

(c) (i) Open Access This article is licensed under a Creative Commons Attribution 4.0 International License, which permits use, sharing, adaptation, distribution and reproduction cc) in any medium or format, as long as you give appropriate credit to the original author(s) and the source, provide a link to the Creative Commons license, and indicate if changes were made. The images or other third party material in this article are included in the article's Creative Commons license, unless indicated otherwise in a credit line to the material. If material is not included in the article's Creative Commons license and your intended use is not permitted by statutory regulation or exceeds the permitted use, you will need to obtain permission directly from the copyright holder. To view a copy of this license, visit http://creativecommons.org/licenses/by/4.0/. 
intrinsic mechanisms of SCs self-renewal, proliferation, differentiation, and fusion has vital application value for the manipulation of SCs behaviors in vitro, as well as the restart of muscle regeneration program in vivo.

Previous studies have shown that the functions of SCs are regulated by multiple signal pathways ${ }^{13-15}$. Among them, protein kinase, serving as the extremely important signal proteins in mammalian cells ${ }^{16}$, plays a crucial role in the regulation of SCs functions. For example, protein kinase ERK and JNK can facilitate SCs self-renewal $^{17,18}$, and TAK can promote SCs proliferation and repress differentiation ${ }^{19}$, while p38 MAPK, Lkb1, and JAK can inhibit proliferation and accelerate differentiation of $\mathrm{SCs}^{20-22}$. It can be seen that the effect of these reported protein kinases on SCs is just reflected either in one state of SCs, or in boosting one state but restricting another state. Meanwhile, the proliferation, differentiation, and fusion of muscle SCs during regeneration are a staggered sequential process. Thus, the utilization of these reported protein kinase targets should be paid attention to the selection of the time window during muscle regeneration. Once the application opportunity is not well mastered, it will result in inadequate expansion or premature differentiation in myoblasts, which in turn limits the proper muscle repair. This problem virtually increases the difficulty and risk of clinical application of the reported kinase targets. Therefore, in this paper we will attempt to screen novel protein kinase that benefits multiple states of myoblasts, and reveal its effects on myoblast behaviors and muscle regeneration, providing potential target for the myoblast-based therapeutics of skeletal muscle injury.

\section{Materials and methods Bioinformatics}

An expression profile microarray data of muscle injury model were downloaded from Gene Expression Omnibus (GEO) database. The wild-type control group and wildtype notexin (NTX) treatment group were selected and reanalyzed. The limma $\mathrm{R}$-package of the $\mathrm{R}$ platform was used according to the user's guide for screening differentially expressed genes (DEGs) between these two groups. The DEGs were identified with the following criterion: $\mid$ fold change $\mid \geq 2$ and $P$-value $<0.01$. Then the DEGs were further annotated and the protein kinases among these DEGs were identified by gene ontology (GO) analysis using the Database for Annotation, Visualization, and Integrated Discovery (DAVID) v6.8 (https://david.ncifcrf. gov/). The volcano plot of the DEGs and the heat map of the differentially expressed protein kinases were drawn via the ggplots package in the $\mathrm{R}$ platform. The venn diagram was created through website Draw Venn Diagram (http:// bioinformatics.psb.ugent.be/webtools/Venn/).

\section{Generation of Pim1 knockout mice}

Pim1 knockout $\left(\mathrm{Pim1}^{-1-}\right)$ mice was obtained from Biomodel Organisms (Shanghai, China). Briefly, Pim1 $1^{-1-}$ mice lacking exon I-VI of the Pim1 gene were generated using CRISPR/Cas9-mediated genome editing in C57BL/ 6J embryonic stem cell. Heterozygous animals were bred to obtain homozygous Pim1 $1^{-1-}$ mice. The Pim1 $1^{-1-}$ mouse strain was genotyped by PCR using the primers 5'-CGGCGTTAGCGACCATTCTG-3' and 5'-GGAAGA GGTGACAGGGACTTAA-3'. Mice were housed under specific pathogen-free conditions at $24 \pm 2{ }^{\circ} \mathrm{C}$ with a $12: 12 \mathrm{~h}$ light/dark cycle and ad libitum access to food and water. All animal experimental procedures were in accordance with the National Institutes of Health Guide for the Care and Use of Laboratory Animals and approved by the Ethical Committee for Animal Experimentation of the School of Medicine at Shandong University (Document No. LL-201602035).

\section{Muscle injury}

The muscle injury mouse model was established by intramuscular injection of $10 \mu \mathrm{L}$ of NTX $(10 \mu \mathrm{g} / \mathrm{ml}$, L8104, Latoxan) into the tibialis anterior (TA) muscle under pentobarbital sodium anesthesia $(50 \mathrm{mg} / \mathrm{kg}$, i.p.). The TA muscles were harvested and weighed at different time points post injection and then quick-frozen in liquid nitrogen and stored at $-80^{\circ} \mathrm{C}$ or immersed directly in $4 \%$ paraformaldehyde (PFA) until the time of molecular or histological analysis.

\section{Cell viability assay}

C2C12 cell viability was determined using the CCK- 8 kit in accordance with the manufacturer's protocol. In brief, C2C12 cells were seeded on 96-well cell culture plates at a density of 5000 cells per well. At $24,36,48$, and $60 \mathrm{~h}$ after seeding, $10 \mu \mathrm{L}$ CCK- 8 solution was added to $100 \mu \mathrm{L}$ medium and incubated at $37^{\circ} \mathrm{C}$ for $1 \mathrm{~h}$. Then, the optical densities of the cells at $450 \mathrm{~nm}$ were measured with a Multiskan Mk3 microplate reader (Thermo Scientific). Cell viability was calculated as a proportion of the control group.

\section{Proliferation and apoptosis assays}

To assay proliferation, $\mathrm{C} 2 \mathrm{C} 12$ cell lines were incubated with EdU at a final concentration of $10 \mu \mathrm{M}$ for $2 \mathrm{~h}$ before fixation and stained nuclei with Hoechst using the ClickiT EdU Alexa Fluor 555 Imaging kit (C10638, Invitrogen) according to the manufacturer's protocol. To assay apoptosis, TUNEL staining was performed to monitor the cell apoptosis using the In Situ Cell Death Detection Kit (11684795910, Roche) in accordance with the manufacturer's instructions. Images were then captured using an Olympus BX63 fluorescence microscope. Orange red EdU dots or green TUNEL dots located within the 
nucleus were defined as proliferative or apoptotic cells. The ratio of proliferative or apoptotic cells to total cells was calculated.

\section{Luciferase activity assay}

For the MyoD transcriptional activity assay, $\mathrm{C} 2 \mathrm{C} 12$ myoblasts in 24-well plate were transfected with expression plasmids for Pim1 K67M-Flag (200 ng), MyoD-HA (100 ng), the E-box-specific 4RE-luciferase reporter (4REluc) (300 ng), $\quad M y o G$ promoter $(1444 \mathrm{bp})$-luciferase reporter (MyoG pro-luc) (300 ng) and Renilla luciferase (RL) reporter $(20 \mathrm{ng})$ using lipofectamine 2000 (Invitrogen). Transfected cells were induced to differentiate for $72 \mathrm{~h}$. Then the cells were lysed and luciferase activity was measured for four times by a microplate luminometer (Centro LB 960, Berthold) with the Dual-Glo Luciferase Assay System (Promega). Firefly luciferase activity was normalized to the Renilla luciferase activity for each transfected well and the results were presented as a proportion of the activity of the basic luciferase vector (4REluc).

\section{Muscle force measurement}

The function of TA muscles was evaluated by measuring in vivo muscle contraction in response to nerve stimulation. The mice were anaesthetized with pentobarbital sodium $(50 \mathrm{mg} / \mathrm{kg})$. The knee and paw were fixed in place to prevent movement from the contraction of other muscle groups and the distal tendon of the TA muscle was dissected and attached to a JZ101 tension transducer (Yuyan Instruments, Shanghai, China) using a silk ligature. Electrical stimulations were applied across two needle electrodes placed beneath the TA muscle to stimulate the tibial nerve. Capacity for force generation was evaluated by measuring the absolute maximal force that was generated during tetanic contractions in response to electrical stimulation (train of $55 \mathrm{~Hz}$ for $300 \mathrm{~ms}$ pulses). Data were collected with a Medlab Data Acquisition and Processing Systems (Yuyan Instruments). Force was normalized by muscle mass as an estimate of specific tetanic force.

\section{Statistical analysis}

The statistical significance of differences between experimental groups was determined by a two-tailed Student's $t$-test using SPSS 13.0 software $^{23}$. The data are presented as the mean \pm SD or mean \pm SEM $(* P<0.05$; ${ }^{* *} P<0.01$; and $\left.{ }^{* * *} P<0.001\right)$.

\section{Results}

Pim1 kinase is upregulated after skeletal muscle injury

To screen novel protein kinases that regulate skeletal muscle regeneration, we firstly downloaded the expression profile microarray data GSE67032 from GEO database. The data of wild-type control group and wildtype NTX treatment group were reanalyzed for screening the DEGs by the limma R-package of the R platform. A total of 2703 genes were obtained, including 1634 upregulated and 1069 downregulated (Fig. 1a). According to GO enrichment analysis for biological process and molecular function, we got 89 genes that participate in protein phosphorylation and 77 protein kinases from the 2703 DEGs (Fig. 1b, c). Furthermore, 73 genes were obtained by taking the intersection of these two GO terms (Fig. 1d). Pim1 was one of the upregulated protein kinases modulating protein phosphorylation (Fig. 1e).

Next, we established a skeletal muscle injury mouse model by NTX intramuscular injection to verify the expression of Pim1. As previously described ${ }^{24}$, the $34-\mathrm{kDa}$ isoform of Pim1 was visualized as two bands in whole-cell lysate immunoblots: the phosphorylated band of higher moiety and the unphosphorylated band of lower moiety. In our research, western blot and qPCR showed that both protein and mRNA expression of Pim1 were significantly upregulated in TA muscle at day 3 and day 7 after NTX injection, and returned to normal at day 14 and day 28 post-injection (Fig. 1f, g). Importantly, the time window of Pim1 upregulation in our study coincides with the peak of myoblast proliferation and differentiation ${ }^{5}$, suggesting that Pim1 kinase is likely involved in the process of myoblast mediated-skeletal muscle regeneration.

\section{Pim 1 kinase is required for myoblast proliferation and survival in vitro}

To gain a better understanding of the effect of Pim1 on myoblast behaviors, we established Pim1 low-expression and over-expression $\mathrm{C} 2 \mathrm{C} 12$ myoblast cell lines by LvshPim1 or Lv-Pim1 infection. As expected, infection with Lv-shPim 1 efficiently downregulated, while infection with Lv-Pim1 upregulated Pim1 protein level in C2C12 myoblasts (Fig. 2a). Lv-shPim1 infection inhibited the cell viability in a time-dependent manner, as shown by CCK-8 assay (Fig. 2b). Conversely, Pim1 overexpression enhanced the $\mathrm{C} 2 \mathrm{C} 12$ myoblast viability (Fig. 2c). Meanwhile, the reduced cell viability after Lv-shPim1 infection was accompanied by a marked reduction of cell proliferation, as indicated by a decrease of the number of EdU $^{+}$cell (Fig. 2d, e). Meanwhile, we observed an increase in $\mathrm{TUNEL}^{+}$cell (Fig. 2f, g) in Pim1 lowexpression $\mathrm{C} 2 \mathrm{C} 12$ myoblasts, suggesting that Pim1 knockdown in $\mathrm{C} 2 \mathrm{C} 12$ myoblasts accelerated apoptosis. Altogether, these findings indicated that the proliferation and survival of $\mathrm{C} 2 \mathrm{C} 12$ myoblasts are dependent on Pim1 kinase. Consistent with the results from $\mathrm{C} 2 \mathrm{C} 12$ cell lines, Pim1 knockdown also restrained the proliferation of primary cultured myoblasts as demonstrated by a marked reduction of the proportion of $\mathrm{Pax}^{+}$and $\mathrm{MyoD}^{+}$cell (Fig. 2h-k). 


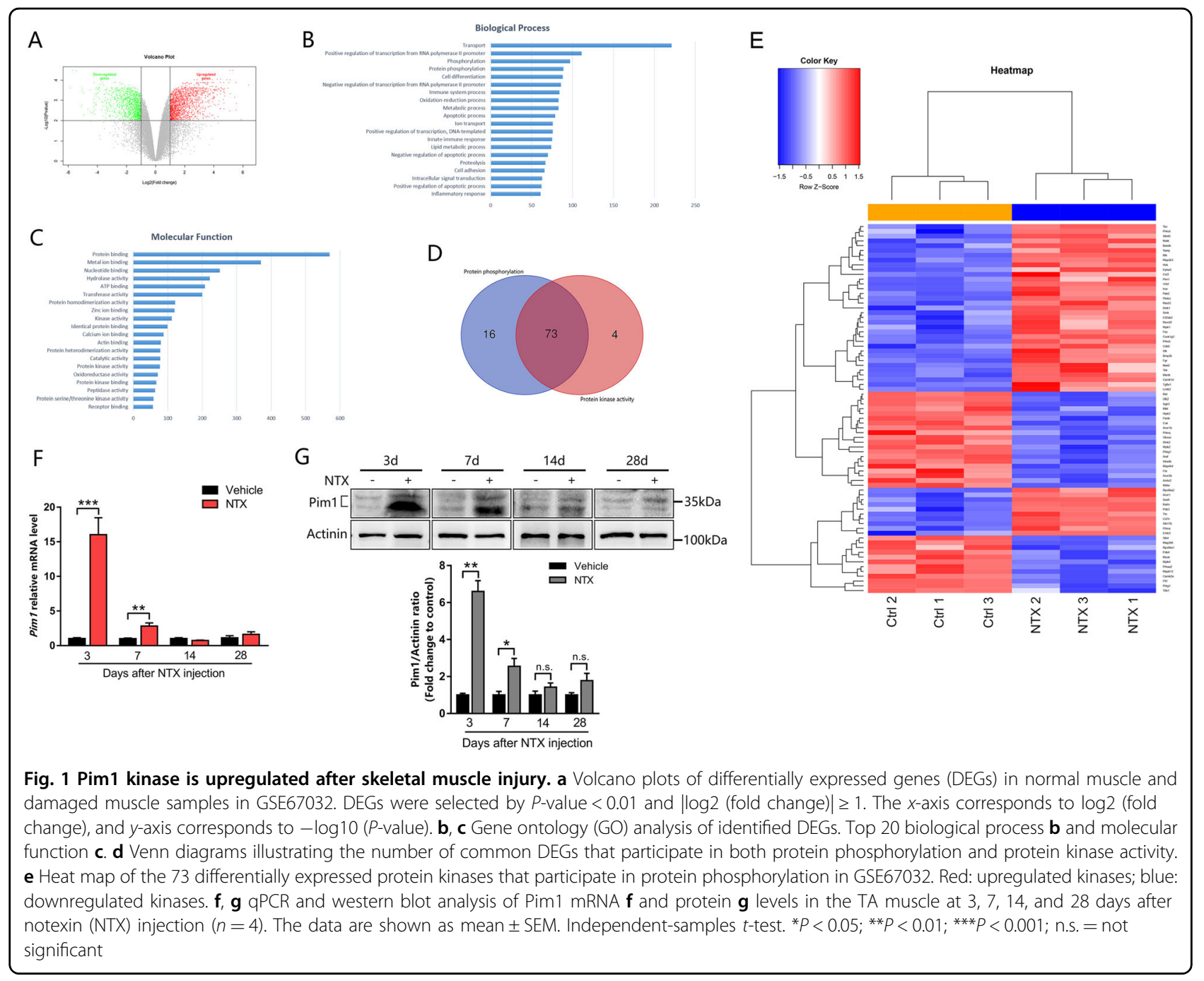

Pim1 kinase is upregulated and translocated from cytoplasm into nucleus during myogenic differentiation

To investigate the association of Pim1 kinase with myoblast differentiation, we first tested the localization of Pim1 kinase in proliferating and differentiated SCs by immunofluorescence. To our surprise, the Pim1 kinase was present in the cytoplasm during SCs proliferation, but was mostly transferred into the nucleus upon differentiation (Fig. 3a). Then, we analyzed the Pim1 expression in whole-cell lysate over the time course of differentiation. Notably, qPCR and western blot showed a gradual increase in Pim 1 mRNA and protein during $\mathrm{C} 2 \mathrm{C} 12$ cell differentiation (Fig. 3b, c). Subcellular fractionation study showed that the cytosolic Pim1 protein increased by 1.9fold, while the nuclear Pim1 protein increased by 2.8 -fold at day 3 post differentiation (Fig. 3d). Importantly, the nuclear Pim1 was visualized as the phosphorylated band of higher moiety in immunoblots while the cytosolic Pim1 was unphosphorylated (Fig. 3d). We further transduced the wild-type Pim1 plasmid and the kinase-inactive Pim1 K67M plasmid into $\mathrm{C} 2 \mathrm{C} 12$ myoblasts. Immunofluorescence showed that the kinase-inactive Pim1 K67M was not transferred into the nucleus during differentiation (Fig. 3e), suggesting that the activity of Pim1 kinase is necessary for its nuclear translocation.

\section{Pim1 kinase in nuclei facilitates myoblast differentiation by mediating MyoD activity}

To further evaluate the requirement of Pim1 kinase activity in nuclei on myoblast differentiation in vitro, we treated $\mathrm{C} 2 \mathrm{C} 12$ myoblasts and primary myoblasts with Pim1 kinase inhibitor TCS during the induction of differentiation. Immunofluorescence showed that inhibition of Pim 1 activity did impair myogenic differentiation and fusion of $\mathrm{C} 2 \mathrm{C} 12$ cells and primary myoblasts, as indicated by a lower differentiation index and fusion index compared to control (Fig. 4a-f). Meanwhile, the expression of myogenic regulatory factor myogenin, muscle structural 


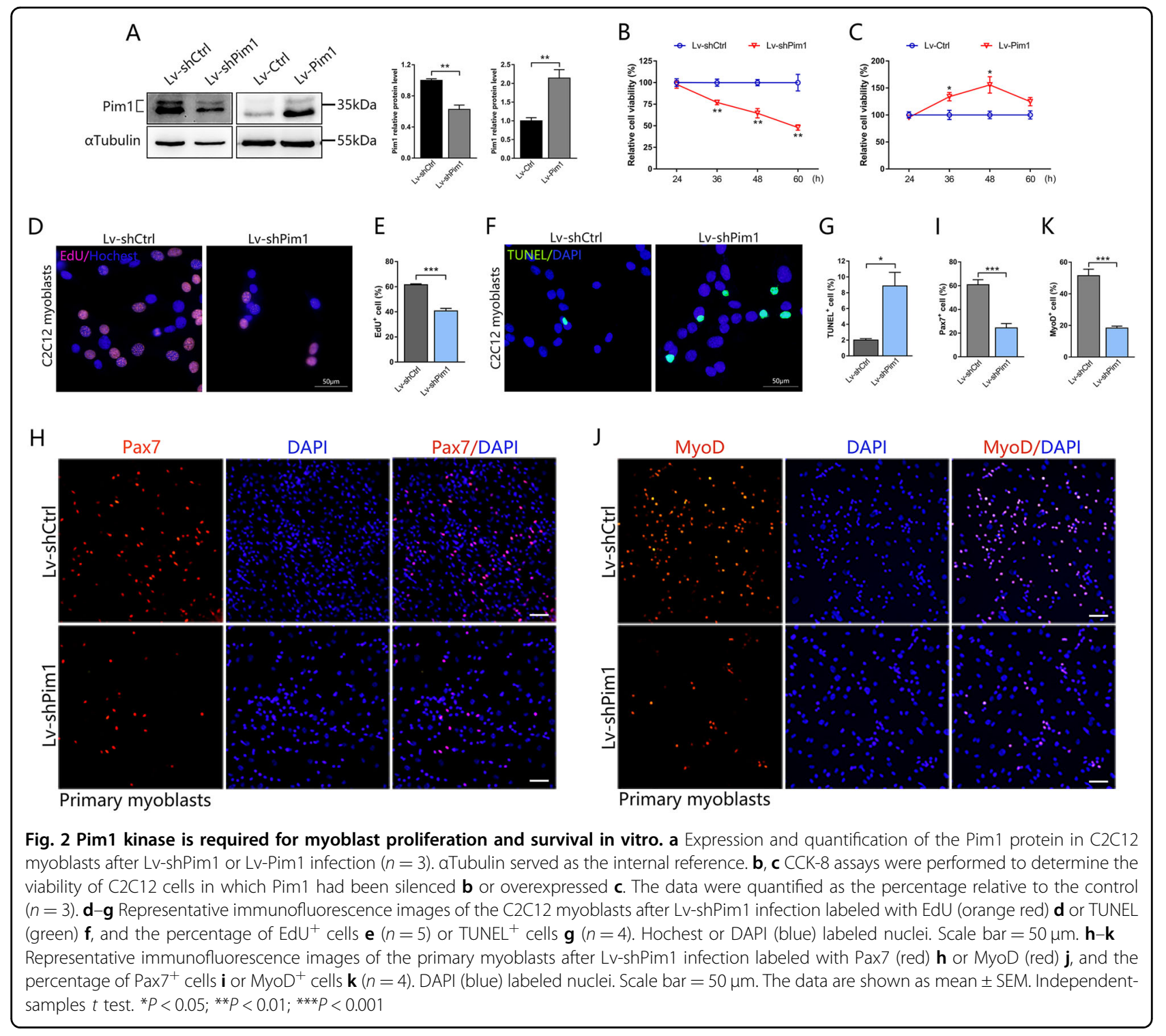

protein $\mathrm{MyHC}$, and fusion-related protein myomerger was restrained upon TCS treatment in a dose-dependent manner (Fig. 4g). Similarly, qPCR results showed a significant decrease in the mRNA level of muscle-specific genes (MyoG, Tnni2, Ckm, Mylpf, Acta1, Mymk, and Mymx) involved in myogenic differentiation and fusion after TCS treatment (Fig. 4h).

To investigate the mechanisms by which Pim1 kinase promotes myogenic differentiation, we focused on MyoD, the basic helix-loop-helix (bHLH) myogenic regulatory factor that drives the specification and differentiation of myoblasts into myotubes ${ }^{25,26}$. Given that the inhibition of Pim1 activity did not reduce the protein expression of MyoD in the differentiated C2C12 cells (Fig. S1), while the mRNA expression of MyoD-targeting genes ( $M y o G, C k m$, Tnni2) was significantly inhibited (Fig. 4h). Therefore, we hypothesized that Pim1 kinase activity contributes to the MyoD-dependent transcriptional activation. To test this hypothesis, we analyzed the activity of 4RE-luc (firefly luciferase reporter plasmid controlled by MyoD-binding core sequence) and $M y o G$ pro-luc (firefly luciferase reporter plasmid controlled by $M y o G$ promoter) by luciferase reporter assay in $\mathrm{C} 2 \mathrm{C} 12$ cells. As shown in Fig. 4i, j, both kinase-inactive Pim1 K67M plasmid and Pim1 inhibitor TCS significantly inhibited the activity of 4REluc and MyoG pro-luc. To explore how Pim1 affects the transcriptional activity of MyoD, we performed coimmunoprecipitation (Co-IP) in $\mathrm{C} 2 \mathrm{C} 12$ cells transfected with Lv-Flag-Pim1. As shown in Fig. 4k, Co-IP experiment of nuclear protein using Flag antibody revealed the interaction between Pim 1 and MyoD on 4 days postdifferentiation. Furthermore, confocal images showed a 


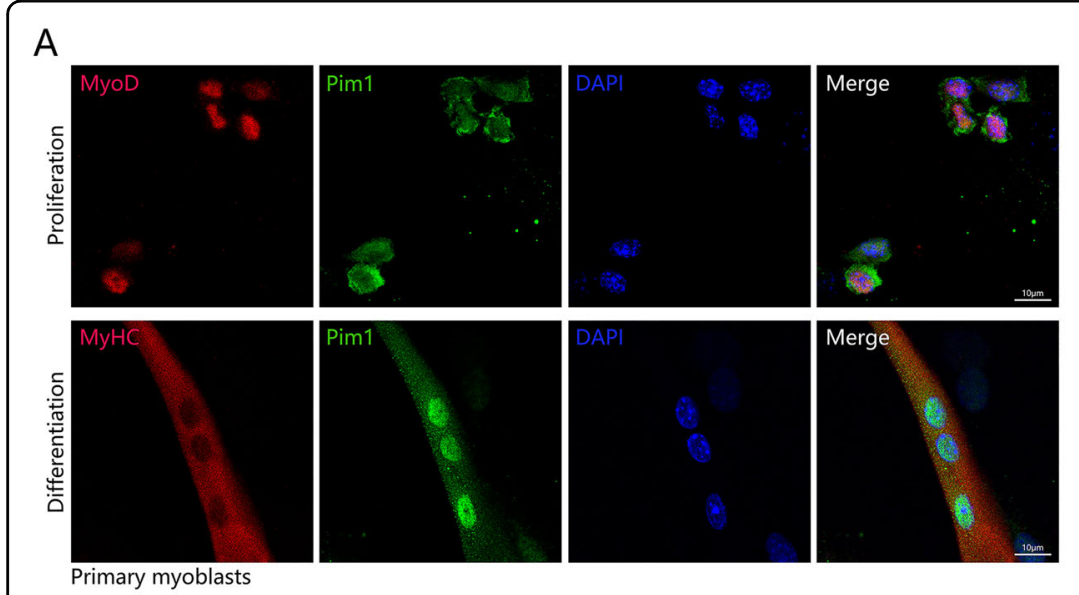

B

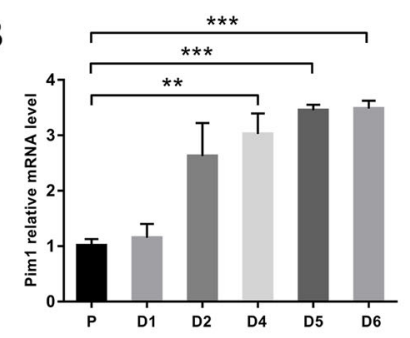

C

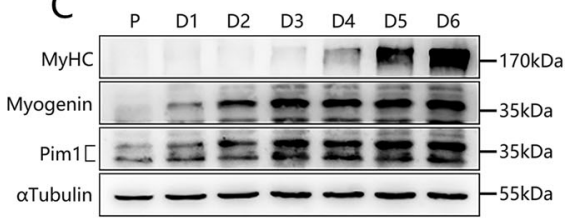

Cytoplasm
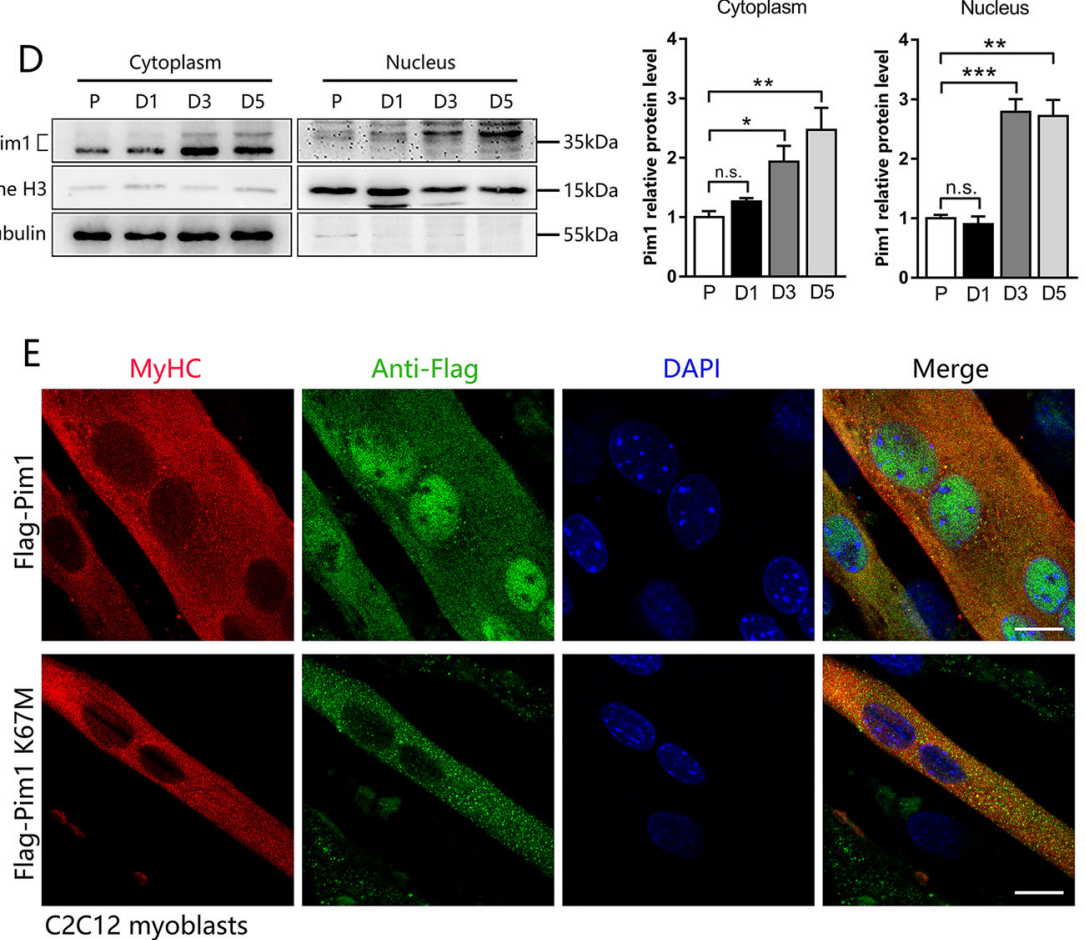

Fig. 3 Pim 1 kinase is upregulated and translocated from cytoplasm into nucleus during myogenic differentiation. a Immunofluorescence was performed to determine the subcellular localization of Pim1 kinase in proliferative and differentiated primary myoblasts. Green for Pim1 kinase; Red for MyoD or MyHC; Blue for nuclei labeled with DAPI. Scale bar $=10 \mu \mathrm{m}$. b, c qPCR and western blot analysis of Pim1 mRNA $\mathbf{b}$ and protein c levels during myogenic differentiation $(n=3)$. P proliferation, D differentiation. $\mathbf{d}$ Subcellular fractionation analysis showing the protein level of cytosolic Pim1 and nuclear Pim1 during myogenic differentiation $(n=4)$. P proliferation, D differentiation. e Confocal images for the analyses of subcellular localization of Pim1 in 5-day-differentiated C2C12 myotubes using anti-Flag (green) and anti-MyHC (red) antibodies. C2C12 myoblasts were transduced with the wild-type Pim1 plasmid and the kinase-inactive Pim1 K67M plasmid, and induced for differentiation. Scale bar $=10 \mu \mathrm{m}$. The data are shown as mean \pm SEM. Independent-samples $t$ test. ${ }^{*} P<0.05$; ${ }^{* *} P<0.01$; ${ }^{* *} P<0.001 ;$ n.s. $=$ not significant

strong colocalization between Pim1 and MyoD in the nucleus of $\mathrm{C} 2 \mathrm{C} 12$ and primary myotube (Fig. 4l, S2). Taken together, these data suggested that Pim1 kinase combines with myogenic regulator MyoD in myonuclei and facilitates its transcriptional activity, inducing the expression of muscle-specific genes, which consequently promotes myogenic differentiation.
Pim $1^{-1-}$ mice exhibit no myopathy but a deficit of muscle regeneration

We generated Pim1 knockout $\left(\operatorname{Pim} 1^{-1-}\right)$ mice through the ablation of exon I-VI of Pim1 gene by using CRISPR/Cas9-mediated gene editing system (Fig. 5a). All mice were viable and were identified by tail genotyping (Fig. 5b). Firstly, we analyzed the body weight, 


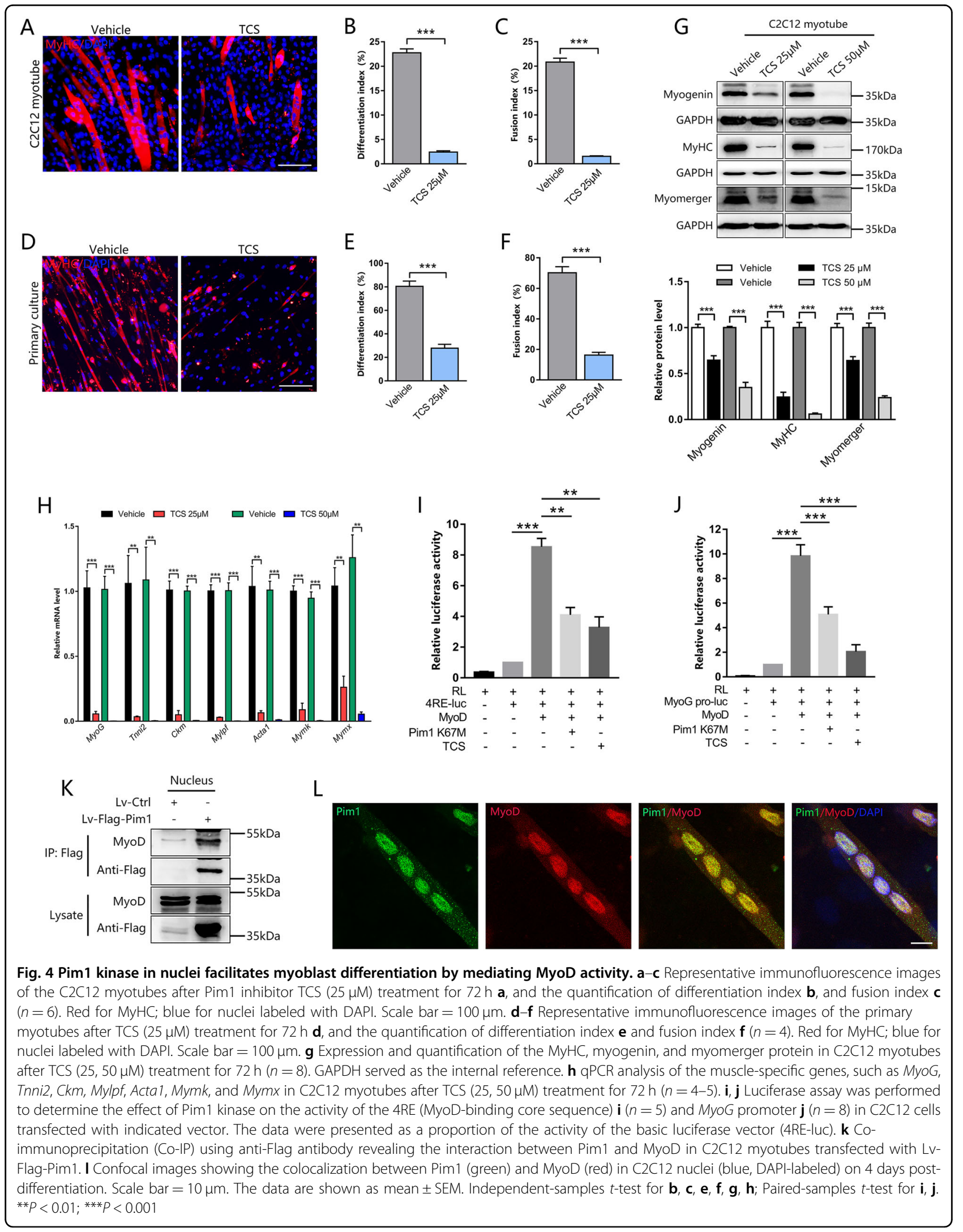




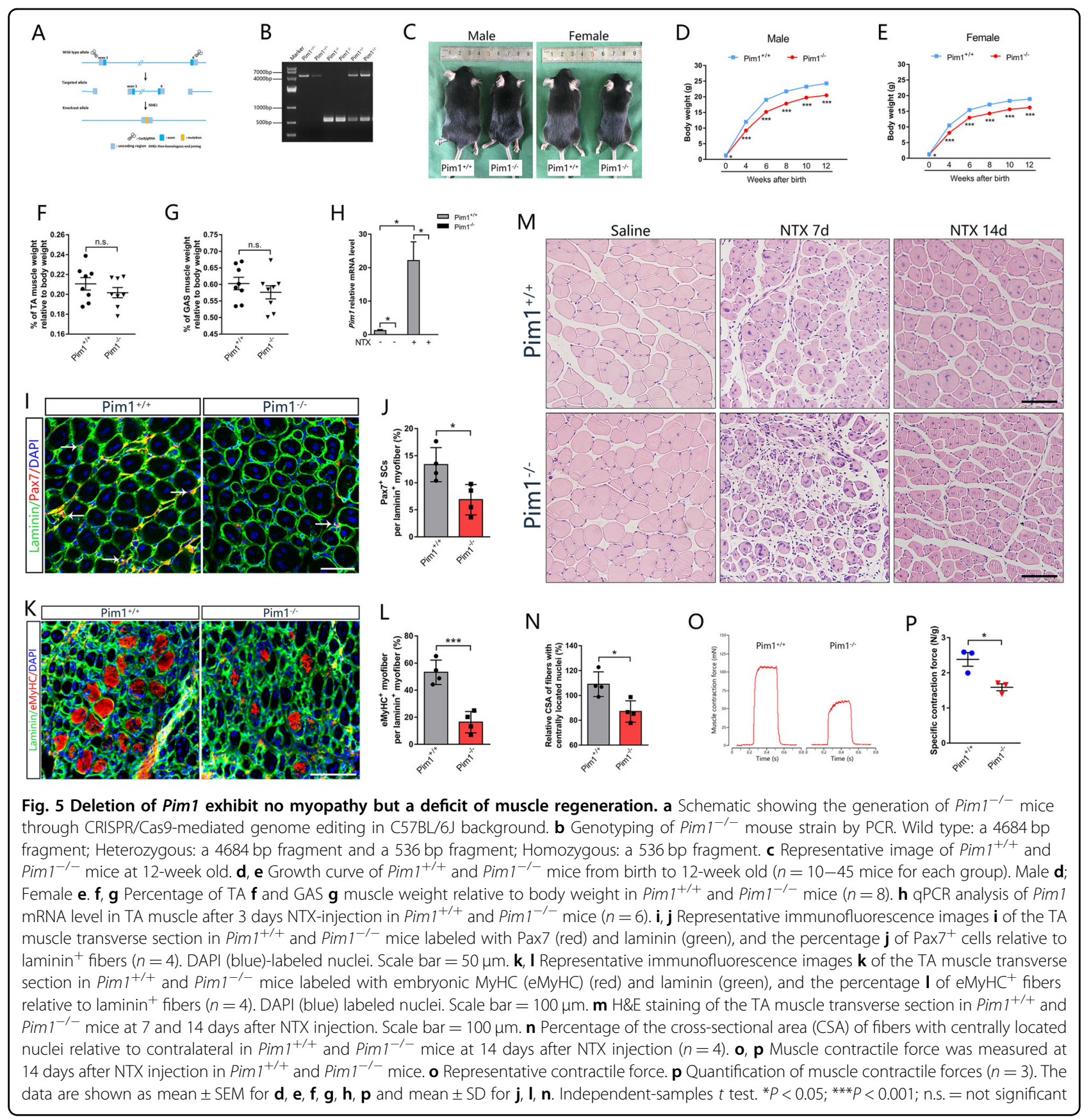

muscle weight, and morphology of $P i m 1^{-1-}$ mice and wild type $\left(P i m 1^{+/+}\right)$mice in age-matched groups. In a 12-week follow-up, the body weight of Pim $1^{-1-}$ mice was significantly lower than that of control littermates (Fig. $5 \mathrm{c}-\mathrm{e}$ ), showing an overall growth retardation. But the proportion of the wet weight of TA and GAS muscles relative to body weight was not different from that of control littermates (Fig. 5f, g). H\&E staining also showed that Pim $1^{-1-}$ mice has no severe myopathy characterized by central nucleated myofibers from birth to 12 -week old (Fig. S3).
To determine whether deletion of Pim1 affects skeletal muscle regeneration in vivo, we established a NTXinduced muscle injury model with 10-12-week-old Pim $1^{+/+}$and Pim1 $1^{-/-}$male mice. Strikingly, a virtually complete absence of the mRNA and protein of Pim1 was observed in isolated TA muscles from both saline and NTX treated Pim1 $1^{-1-}$ mice (Fig. 5h, S4), which ensured that Pim1 gene was deleted in TA muscle. Firstly, the SCs in Pim $1^{-1-}$ mice showed a striking block of cell expansion 7 days after injury, as reflected by a marked reduction of the number of $\mathrm{Pax}^{+}$cell $\left(\operatorname{Pim} 1^{+/+} 13.34 \pm 3.15 \%\right.$, Pim $^{-/-}$ 
$6.86 \pm 2.81 \%$ ) (Fig. 5i, j). The frequency of embryonic $\mathrm{MyHC}$ (eMyHC, an early marker of muscle regeneration) positive myofibers within laminin staining was dramatically decreased in TA muscle of Pim1 $1^{-1-}$ mice at 5 days post injury (Fig. 5k, l). Next, we performed histological methods to analyze the effect of Pim1 on muscle fiber morphology during regeneration. Compared with $\mathrm{Pim}^{+/+}$ littermates, Pim $1^{-1-}$ mice showed an impaired muscle regeneration, as indicated by a smaller CSA of fibers with centrally located nuclei at 14 days post injury (Fig. $5 \mathrm{~m}, \mathrm{n}$ ). These changes reflect the remodeling of muscle architecture that accompanies the retarded regeneration in Pim $1^{-/-}$mice, as the relative weight of injured TA muscle in $P i m 1^{-1-}$ mice did not decrease during this time period (Fig. S5). In addition to morphology, we then investigated whether the impaired myofiber regeneration in Pim1deficient mice altered the function of muscle contraction. We observed a marked reduction in muscle contraction as demonstrated by a striking decrease in specific tetanic force in the damaged TA muscle of Pim $1^{-l-}$ mice at day 14 after injury (Fig. 5o, p). Altogether, these findings showed that deletion of Pim1 in skeletal muscle restricts myofiber regeneration morphologically and retards strength recovery functionally upon muscle injury, emphasizing the requirement of Pim1 for proper skeletal muscle repair.

\section{Discussion}

Pim1, a serine/threonine protein kinase, is a member of the proviral integration site for moloney murine leukemia virus (Pim) kinase family composed of three different isoforms (Pim1, Pim2, Pim3) ${ }^{27}$. According to molecular weight and cellular localization, Pim1 kinase contains two isoforms, a long $44 \mathrm{kDa}$ isoform, located in cell membrane and a short $34 \mathrm{kDa}$ isoform, localized in cytoplasm and nucleus $^{28,29}$. Pim1 kinase is constitutively active when expressed in cells, and its activity is directly correlated with its expression level. Additionally, the posttranslational modification such as phosphorylation or dephosphorylation also affects Pim1 activity ${ }^{29,30}$.

Functionally, studies have well proven that the $34 \mathrm{kDa}$ Pim1 plays a crucial role in a wide range of cell processes and diseases, such as cell proliferation, apoptosis, mitochondrial integrity, cellular senescence, myocardial injury, lupus nephritis and Alzheimer's disease through interaction, stabilization, and phosphorylation of many downstream targets ${ }^{29,31-38}$. Importantly, as a direct target gene of JAK/STAT-signaling pathway ${ }^{21,39,40}$, Pim1 kinase is also involved in immune regulation and inflammatory response. Pro-inflammatory factors enhance Pim1 expression, and inhibition of Pim 1 activity reduces the production of inflammatory factors and chemokines ${ }^{41}$. Furthermore, inflammation engages in almost all biological processes in mammals. So Pim1 kinase exerts its effects likely by mediating inflammatory response. Meanwhile, emerging evidences demonstrate that muscle regeneration after injury entail appropriate immune responses and inflammatory signals that orchestrate efficacious muscle SCs functions ${ }^{7,19,21,42-45}$. Consequently, based on the above reasoning, we hypothesized that Pim1 kinase may be an important modulator for SCs-mediated skeletal muscle regeneration.

In this study, we found that Pim1 kinase is upregulated in the process of muscle regeneration through the expression profile DEGs screening between normal muscle and injured muscle, bioinformatics analysis, and experimental verification of muscle injury mouse model. This upregulation occurs on day 3 and 7 after muscle injury. Interestingly, evidence has demonstrated that the production of many inflammatory factors and chemokines in skeletal muscle is also increased sharply within 3 days after injury ${ }^{7,42,44}$. So we speculated that Pim1 upregulation after muscle injury may be caused by the stimulation of inflammatory factors and the activation of JAK/STATsignaling pathway, but this inference needs further experimental evidence. More importantly, the peak of SCs proliferation is also at 3-4 days post-injury, then SCs enter the stage of differentiation and fusion at 6-14 days post-injury ${ }^{5}$. It can be seen that the time window of Pim1 upregulation coincides with the peak period of SCs proliferation and differentiation, suggesting that Pim1 kinase is likely involved in SCs myogenic lineage progression.

Next, we established muscle injury model on $P i m 1^{-1-}$ mice to investigate the role of Pim1 kinase in skeletal muscle regeneration. It was found that deletion of Pim1 shows a significant decrease in the CSA of muscle fibers with centrally located nuclei, implying that Pim 1 kinase is vital for normal muscle regeneration. Since SCs are the ultimate executors of myofiber regeneration, we further explored the effects of Pim1 kinase on SCs behaviors at various stages. Consistent with the key role of Pim1 kinase in promoting proliferation and inhibiting apoptosis, it was found in this paper that Pim1 knockdown significantly restrained the proliferation and accelerated the apoptosis of myoblasts in vitro, indicating the necessity of Pim1 for myoblast survival and amplification (Fig. 6). In addition, we found that the expression level and subcellular localization of Pim1 changes over the course of myogenesis. Pim1 predominantly localizes to the cytoplasm of proliferating myoblast, but it is upregulated and translocated to the nucleus upon differentiation (Fig. 6). Moreover, in differentiated myotube, the nuclear Pim1 is phosphorylated while the cytosolic Pim1 is unphosphorylated. Coincidentally, a recent study has highlighted that the functional effect of Pim1 depends upon intracellular localization in human cardiac progenitor cells ${ }^{46}$. Thus, these evidences suggested that the upregulation of phosphorylated Pim1 in myocyte nucleus is likely associated 


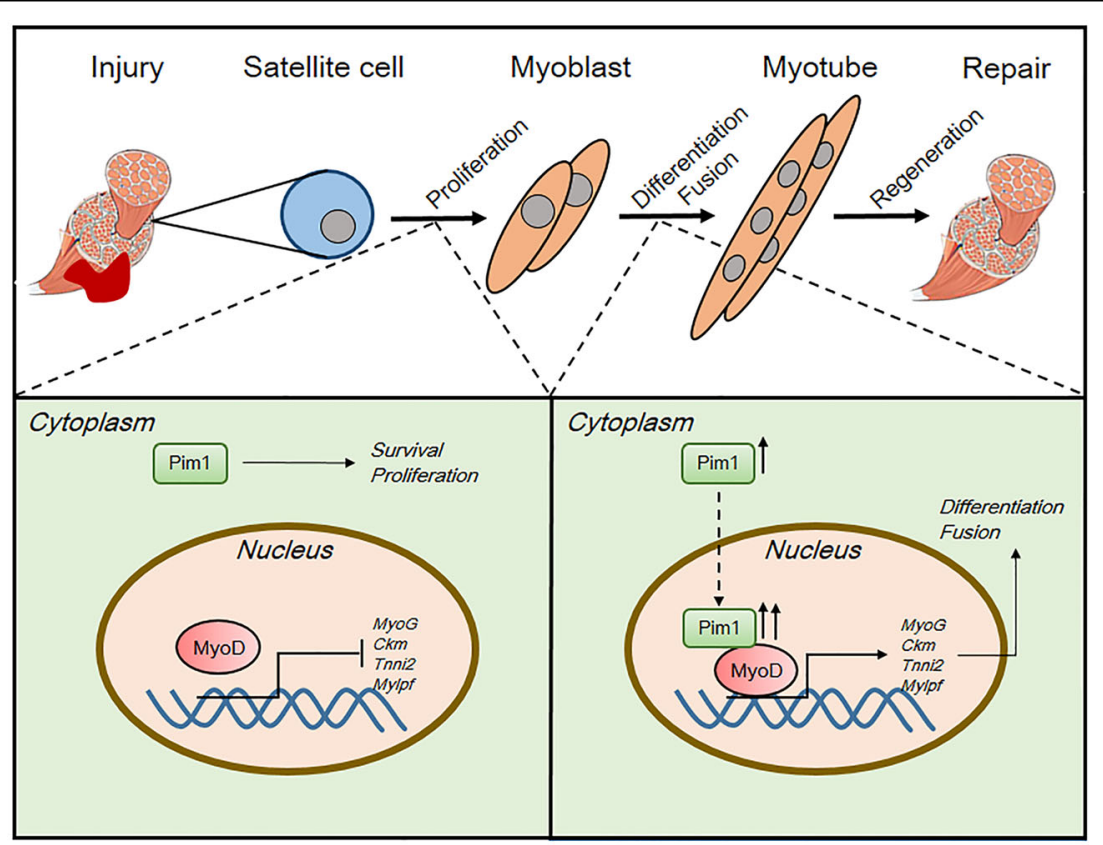

Fig. 6 Schematic showing the regulation patterns of Pim1 kinase on myoblast and MyoD transcriptional activity during muscle regeneration. First, Pim1 kinase is required for myoblast survival and proliferation. Second, Pim1 kinase is upregulated and translocated from cytoplasm into nucleus during myogenic differentiation. Third, Pim1 kinase interacts with myogenic regulator MyoD, controls the transcriptional activity of MyoD, inducing the expression of muscle-specific genes, which consequently promotes the myogenic differentiation and fusion

with myogenic differentiation. By using Pim1 kinase inhibitor, we proved that inhibition of Pim1 activity prevents myoblast differentiation and fusion, suggesting that Pim1 kinase activity is required for the proper myogenesis (Fig. 6).

With respect to the underlying mechanism, previous study has confirmed that Pim1 contributes to bHLH transcription factor c-Myc-dependent transcriptional activation $^{47}$. Interestingly, myogenic regulator $\mathrm{MyoD}$ is also a transcription factor with bHLH domain ${ }^{25,26}$. And, the direct targets of MyoD are downregulated upon Pim1 inhibition in our study. Therefore, we speculated that Pim1 kinase facilitates myoblast differentiation likely by mediating the transcriptional activity of MyoD. By Co-IP experiment and luciferase activity assay, we showed that Pim1 interacts with the key myogenic regulator MyoD, and either the transfection of kinase-inactive Pim1 plasmid, or the application of Pim1 inhibitor significantly inhibited the MyoDdependent transcriptional activation, suggesting that Pim1 kinase activity facilitates myoblast differentiation to some extent by controlling the transcriptional activity of the bHLH myogenic regulator MyoD.

In summary, we demonstrated for the first time that Pim1 kinase is a positive modulator of myoblast functions and skeletal muscle regeneration, which will provide new ideas and experimental basis for the myoblast-based therapeutic strategies of skeletal muscle injury.

\section{Acknowledgements}

This work was supported by the National Natural Science Foundation of China (No. 81371917).

\section{Author details}

'Department of Anatomy, Shandong University School of Basic Medical Sciences, Jinan 250012, China. ${ }^{2}$ Department of Spine Surgery, Shenzhen People's Hospital, Second Clinical Medical College of Jinan University, Shenzhen 518020, China. ${ }^{3}$ Department of Orthopaedics, Shandong University Qilu Hospital, Jinan 250012, China

\section{Author contributions}

Y.L. conceived the study, designed and carried out the experiments, analyzed the data and wrote the manuscript. Y.S. and Z.Y. were involved in performing animal experiments and collecting data. Z.W. was involved in the data collection. H.L. contributed to the data analysis and interpretation. Z.L. contributed to the data analysis. Z.z.L. provided guidance and financial support throughout the process, and participated in manuscript writing and final approval of the manuscript.

\section{Conflict of interest}

The authors declare that they have no conflict of interest.

\section{Publisher's note}

Springer Nature remains neutral with regard to jurisdictional claims in published maps and institutional affiliations.

Supplementary Information accompanies this paper at (https://doi.org/ 10.1038/s41419-019-1993-3).

Received: 27 April 2019 Revised: 18 July 2019 Accepted: 17 September 2019

Published online: 10 October 2019 


\section{References}

1. Xiong, G. et al. The PERK arm of the unfolded protein response regulates satellite cell-mediated skeletal muscle regeneration. Elife 6, e22871 (2017).

2. Judson, R. N. et al. Inhibition of methyltransferase Setd7 allows the in vitro expansion of myogenic stem cells with improved therapeutic potential. Cell Stem Cell 22, 177-190 (2018).

3. Gerli, M. F. M. et al. Combined Notch and PDGF signaling enhances migration and expression of stem cell markers while inducing perivascular cell features in muscle satellite cells. Stem Cell Rep. 12, 461-473 (2019).

4. Dumont, N. A., Wang, Y. X. \& Rudnicki, M. A. Intrinsic and extrinsic mechanisms regulating satellite cell function. Development 142, 1572-1581 (2015).

5. Theret, M. et al. AMPKa1-LDH pathway regulates muscle stem cell self-renewal by controlling metabolic homeostasis. EMBO J. 36, 1946-1962 (2017).

6. Cosgrove, B. D. et al. Rejuvenation of the muscle stem cell population restores strength to injured aged muscles. Nat. Med. 20, 255-264 (2014).

7. Ho, A. T. V. et al. Prostaglandin E2 is essential for efficacious skeletal muscle stem-cell function, augmenting regeneration and strength. Proc. Natl Acad. Sci. USA 114, 6675-6684 (2017).

8. Périé, S. et al. Autologous myoblast transplantation for oculopharyngeal muscular dystrophy: a phase I/lla clinical study. Mol. Ther. 22, 219-225 (2014).

9. Quarta, M. et al. An artificial niche preserves the quiescence of muscle stem cells and enhances their therapeutic efficacy. Nat. Biotechnol. 34, 752-759 (2016).

10. Briggs, D. \& Morgan, J. E. Recent progress in satellite cell/myoblast engraftment-relevance for therapy. FEBS J. 280, 4281-4293 (2013).

11. Partridge, T. Myoblast transplantation. Neuromuscul. Disord. 12, S3-S6 (2002)

12. Rinaldi, F. \& Perlingeiro, R. C. Stem cells for skeletal muscle regeneration: therapeutic potential and roadblocks. Transl. Res. 163, 409-417 (2014).

13. Baghdadi, M. B. \& Tajbakhsh, S. Regulation and phylogeny of skeletal muscle regeneration. Dev. Biol. 433, 200-209 (2018).

14. Hindi, S. M., Tajrishi, M. M. \& Kumar, A. Signaling mechanisms in mammalian myoblast fusion. Sci. Signal. 6, re2 (2013).

15. Sacco, A. \& Puri, P. L. Regulation of muscle satellite cell function in tissue homeostasis and aging. Cell Stem Cell 16, 585-587 (2015).

16. García-Aranda, M. \& Redondo, M. Protein kinase targets in breast cancer. Int. J. Mol. Sci. 18, E2543 (2017).

17. Hindi, S. M. \& Kumar, A. TRAF6 regulates satellite stem cell self-renewal and function during regenerative myogenesis. J. Clin. Invest. 126, 151-168 (2016).

18. Shi, $\mathrm{H}$. et al. Improved regenerative myogenesis and muscular dystrophy in mice lacking Mkp5. J. Clin. Invest. 123, 2064-2077 (2013).

19. Ogura, Y. et al. TAK1 modulates satellite stem cell homeostasis and skeletal muscle repair. Nat. Commun. 6, 10123 (2015).

20. Brien, P., Pugazhendhi, D., Woodhouse, S., Oxley, D. \& Pell, J. M. p38a MAPK regulates adult muscle stem cell fate by restricting progenitor proliferation during postnatal growth and repair. Stem Cells 31, 1597-1610 (2013).

21. Price, F. D. et al. Inhibition of JAK-STAT signaling stimulates adult satellite cell function. Nat. Med. 20, 1174-1181 (2014).

22. Shan, T. et al. Lkb1 is indispensable for skeletal muscle development, regeneration, and satellite cell homeostasis. Stem Cells 32, 2893-2907 (2014).

23. Liu, Y., Li, J., Shang, Y., Guo, Y. \& Li, Z. CARM1 contributes to skeletal muscle wasting by mediating FoxO3 activity and promoting myofiber autophagy. Exp. Cell Res. 374, 198-209 (2019)

24. Muraski, J. A. et al. Pim-1 regulates cardiomyocyte survival downstream of Akt. Nat. Med. 13, 1467-1475 (2007).

25. Chen, $X$. et al. Malat1 regulates myogenic differentiation and muscle regeneration through modulating MyoD transcriptional activity. Cell Discov. 3, 17002 (2017).
26. $Y u, X$. et al. Long non-coding RNA Linc-RAM enhances myogenic differentiation by interacting with MyoD. Nat. Commun. 8, 14016 (2017).

27. Mary Photini, S. et al. PIM kinases 1,2 and 3 in intracellular LIF signaling, proliferation and apoptosis in trophoblastic cells. Exp. Cell Res. 359, 275-283 (2017).

28. Herzog, S. et al. Pim1 kinase is upregulated in glioblastoma multiforme and mediates tumor cell survival. Neuro Oncol. 17, 223-242 (2015).

29. Warfel, N. A. \& Kraft, A. S. PIM kinase (and Akt) biology and signaling in tumors. Pharmacol. Ther. 151, 41-49 (2015).

30. Fischer, K. M. et al. Pim-1 kinase inhibits pathological injury by promoting cardioprotective signaling. J. Mol. Cell. Cardiol. 51, 554-558 (2011).

31. Banerjee, S. et al. EBNA3C augments Pim-1 mediated phosphorylation and degradation of p21 to promote B-cell proliferation. PLoS Pathog. 10, e1004304 (2014).

32. Din, S. et al. Pim-1 preserves mitochondrial morphology by inhibiting dynamin-related protein 1 translocation. Proc. Natl Acad. Sci. USA 110, 5969-5974 (2013).

33. Fu, R. et al. Pim-1 as a therapeutic target in human lupus nephritis. Arthritis Rheumatol. 71, 1308-1318 (2019).

34. Gao, Y. et al. Pim-1 mediated signaling during the process of cardiac remodeling following myocardial infarction in ovine hearts. J. Mol. Cell. Cardiol. 63 89-97 (2013).

35. Hu, S. et al. Hypoxic preconditioning increases survival of cardiac progenitor cells via the pim-1 kinase-mediated anti-apoptotic effect. Circ. J. 78, 724-731 (2014).

36. Velazquez, R., Shaw, D. M., Caccamo, A. \& Oddo, S. Pim1 inhibition as a novel therapeutic strategy for Alzheimer's disease. Mol. Neurodegener. 11, 52 (2016).

37. Yang, J. et al. PIM1 induces cellular senescence through phosphorylation of UHRF1 at Ser311. Oncogene 36, 4828-4842 (2017).

38. Zhu, N. et al. Pim-1 kinase phosphorylates cardiac Troponin I and regulates cardiac myofilament function. Cell. Physiol. Biochem. 45, 2174-2186 (2018)

39. Gillinder, K. R. et al. Direct targets of pSTAT5 signalling in erythropoiesis. PLoS One 12, e0180922 (2017).

40. Jin, B. et al. PIM-1 modulates cellular senescence and links IL-6 signaling to heterochromatin formation. Aging Cell 13, 879-889 (2014).

41. Lim, R., Barker, G. \& Lappas, M. Inhibition of PIM1 kinase attenuates inflammation-induced pro-labour mediators in human foetal membranes in vitro. Mol. Hum. Reprod. 23, 428-440 (2017).

42. Chazaud, B. Inflammation during skeletal muscle regeneration and tissue remodeling: application to exercise-induced muscle damage management Immunol. Cell Biol. 94, 140-145 (2016).

43. Hindi, S. M. et al. MyD88 promotes myoblast fusion in a cell-autonomous manner. Nat. Commun. 8, 1624 (2017).

44. Tidball, J. G. Regulation of muscle growth and regeneration by the immune system. Nat. Rev. Immunol. 17, 165-178 (2017).

45. Zhang, C. et al. Complement C3a signaling facilitates skeletal muscle regeneration by regulating monocyte function and trafficking. Nat. Commun. 8 2078 (2017).

46. Samse, $K$ et al. Functional effect of Pim1 depends upon intracellular localization in human cardiac progenitor cells. J. Biol. Chem. 290, 13935-13947 (2015).

47. Zippo, A., De Robertis, A., Serafini, R. \& Oliviero, S. PIM1-dependent phosphorylation of histone $\mathrm{H} 3$ at serine 10 is required for MYC-dependent transcriptional activation and oncogenic transformation. Nat. Cell Biol. 9, 932-944 (2007) 